\title{
INFLUENCE OF A LIQUID ON THE NATURAL FREQUENCIES OF ALMOST CIRCULAR PLATES
}

\author{
MANUEL GASCÓN-PÉREZ* and PABLO GARCÍA-FOGEDA ${ }^{\dagger}$ \\ Escuela de la Ingeniería Aeronáutica y del Espacio \\ UPM, 28040 Madrid, Spain \\ *manuel.gascon@upm.es \\ ${ }^{\dagger}$ pablo.garciafogeda@upm.es
}

\begin{abstract}
In this work, the influence of the surrounding fluid on the dynamic characteristics of almost circular plates is investigated. First the natural frequencies and normal modes for the plates in vacuum are calculated by a perturbation procedure. The method is applied for the case of elliptical plates with a low value of eccentricity. The results are compared with other available methods for this type of plates with good agreement. Next, the effect of the fluid is considered. The normal modes of the plate in vacuum are used as a base to express the vibration mode of the coupled plate-fluid system. By applying the Hankel transformation the nondimensional added virtual mass 2 increment (NAVMI) are calculated for elliptical plates. Results of the NAVMI factors and the effect of the fluid on the natural frequencies are given and it is shown that when the eccentricity of the plate is reduced to zero (circular plate) the known results of the natural frequencies for circular plates surrounded by liquid are recovered.
\end{abstract}

Keywords: Elliptical plate; fluid-structure; natural frequencies; fluid influence.

\section{Introduction}

The interaction of a fluid with a vibrating plate is a problem with many applications in mechanical engineering. When the fluid is air the dynamic characteristics of very light plates used for example in space applications are greatly affected (see Filippi et al. [1994]), in this case also the vibroacoustic properties of the coupled fluid-plate are of great interest [Geng and Li, 2012 or Zheng and Wei, 2013].

When the fluid is a liquid the dynamic properties of the plate are affected even when the plate is not a light structure. Many applications of this interference problem can be found in the literature like the recent works of Tariverdilo et al. [2013] and Askari et al. [2013] that investigate how the vibration of circular plates immersed in a fluid is modified by the depth of immersion. 
For circular plates immersed in a large reservoir of liquid other theoretical and numerical studies can be found in the literature depending on the plate boundary condition. Thus, to cite some of the most classical works for example Rayleigh [1945] calculated the inertial increase of a rigid disk vibrating in a circular aperture; Lamb [1920] obtained the natural frequencies of a clamped circular plate; McLachlan [1932] considered the case of a circular plate with free edges; and Peake and Thurston [1954] considered the cases of clamped and simply supported edges for circular plates. In all of these works, the mode of vibration of the plate was assumed to be a simple polynomial form that in some cases does not satisfy all of the boundary conditions at the plate edges. It can be said, then, that the results in terms of the natural frequencies depend on the choice of the function used to describe each mode shape; therefore, a unique solution is not available.

In recent works, Kwak [1991]; Kwak and Kim [1991]; Amabili et al. [1995] and Amabili and Kwak [1996] have developed a method to determine the dynamic properties of circular plates surrounded by a liquid. The modes of vibration in all of the cases studied were taken as the modes of the plate in vacuum (see, e.g., Leissa [1969]), and these modes satisfy the exact boundary conditions at the plate edges. In Kwak [1991] and Kwak and Kim [1991], the influence of the free surface of the fluid compared with the rigid wall was investigated, and it was concluded that for the cases of clamped and simply supported boundary conditions, the nondimensional added virtual mass increment (NAVMI) factors were lower for the free surface than for the rigid surface. Additionally, in Amabili and Kwak [1996] the influence of the coupling fluid-structure on the vibration mode was studied. In the results presented, it was shown that small differences occur with regard to the mode shape in both cases, with the small deviation depending on the boundary condition on the plate edges. It was concluded in Amabili and Kwak [1996] that the use of the modes in vacuum for the plate vibration can be a good approximation for most of the cases. Therefore, in this paper, the consideration that the plate is in a hole on a rigid surface will be taken into account while keeping in mind that this condition will be different from the case of a plate on a free surface.

For applications to spacecraft structures (such as satellite antennas), the plate shape is not circular but can be considered to be almost circular. Some of the antennas have an elliptical shape with a small ellipticity eccentricity. Thus, by a perturbation procedure the work of Amabili and Kwak [1996], has been extended in the present paper to determine the influence of the fluid on the dynamic characteristics of almost circular plates. First, by applying Lindstedt-Poincaré's technique, the natural frequencies and mode shapes of a clamped elliptical plate in vacuum are obtained. The results of the natural frequencies are compared with other approximations for these types of plates as a function of the eccentricity. Good comparisons are obtained in all of the cases computed. Next, the nondimensional added virtual mass coefficients are computed using the Hankel transformation procedure as used by Amabili and Kwak [1996]. A study of the influence of the added mass as a 
function of the elliptical eccentricity of the plate for different frequencies is presented. When the perturbation parameter $\varepsilon$ is set to zero, the results of Amabili and Kwak [1996] are recovered.

\section{Natural Frequencies of Almost Circular Plates in Vacuum}

Consider a plate vibrating in vacuum. The governing equation describing the plate's amplitude of vibration in the normal direction to the plate surface is

$$
D \nabla^{4} w(r, \theta, t)+\rho_{P} h \frac{\partial^{2} w(r, \theta, t)}{\partial t^{2}}=0,
$$

where $D$ is the flexural rigidity $D=\frac{E h^{3}}{12\left(1-v^{2}\right)}, h$ is the uniform plate thickness, $E$ is the Young modulus of the plate material, $v$ is the Poisson ratio, $\rho_{P}$ is the density of the plate and the Laplacian operator is in polar coordinates:

$$
\nabla^{2}=\frac{\partial^{2}}{\partial r^{2}}+\frac{1}{r} \frac{\partial}{\partial r}+\frac{1}{r^{2}} \frac{\partial^{2}}{\partial \theta^{2}}
$$

If the plate is assumed to be clamped at the edge, the boundary conditions are

$$
\begin{aligned}
w(r, \theta, t)=0 \quad \text { at } r=r(\theta), \\
\frac{\partial w(r, \theta, t)}{\partial n}=0 \quad \text { at } r=r(\theta),
\end{aligned}
$$

where $\mathbf{n}$ is normal to the plate boundary.

If the plate is almost circular with radius $a$, the plate boundary can be expressed in a first approximation as $r=a+\varepsilon f(\theta)$, where $\varepsilon$ is the small parameter of perturbation. Since only linear terms will be considered in this work the present method should only be valid when terms of the order of $\varepsilon^{2}$ can be neglected compared to terms of the order of $\varepsilon$. That is, for example for values of $0<\varepsilon<0.1$ :

The boundary conditions for the plate can then be given as

$$
\begin{aligned}
w(r, \theta, t) & =0, & \text { at } r & =a+\varepsilon f(\theta), \\
\frac{\partial w(r, \theta, t)}{\partial r}-\frac{1}{r^{2}} \varepsilon f^{\prime}(\theta) \frac{\partial w(r, \theta, t)}{\partial \theta} & =0 & \text { at } r & =a+\varepsilon f(\theta) .
\end{aligned}
$$

To search for the normal modes and natural frequencies, the plate is assumed to be in harmonic vibration at the frequency $\omega$, making $w(r, \theta, t)=\tilde{w}(r, \theta) e^{i \omega t}$. The differential equation is now

$$
D \nabla^{4} \tilde{w}(r, \theta)-\rho_{P} \omega^{2} h \tilde{w}(r, \theta)=0 .
$$

Naming

$$
\frac{\rho_{p} h}{D} \omega^{2}=\beta^{4} .
$$

The differential equation is expressed

$$
\nabla^{4} \tilde{w}(r, \theta)-\beta^{4} \tilde{w}(r, \theta)=0 .
$$


And the boundary conditions are

$$
\begin{aligned}
\tilde{w}(r, \theta) & =0 \quad & \text { at } r & =a+\varepsilon f(\theta), \\
\frac{\partial \tilde{w}(r, \theta)}{\partial r}-\frac{1}{r^{2}} \varepsilon f^{\prime}(\theta) \frac{\partial \tilde{w}(r, \theta)}{\partial \theta} & =0 & \text { at } r & =a+\varepsilon f(\theta) .
\end{aligned}
$$

$\tilde{w}(r, \theta)$ represents the amplitude of vibration at $(r, \theta)$.

Applying the method of Lindstedt-Poincare [Nayfeh, 1981], the plate vibration amplitude can be expanded as

$$
\tilde{w}(r, \theta)=\tilde{w}_{0}(r, \theta)+\varepsilon \tilde{w}_{1}(r, \theta)+O\left(\varepsilon^{2}\right) .
$$

And to account for the dependency of the natural frequencies with the perturbation parameter they can be expressed as,

$$
\omega=\omega_{0}+\varepsilon \omega_{1}+O\left(\varepsilon^{2}\right) .
$$

Taking into account the expression $\frac{\rho_{p} h}{D} \omega^{2}=\frac{\rho_{p} h}{D}\left(\omega_{0}^{2}+2 \varepsilon \omega_{0} \omega_{1}+\cdots\right)=\beta^{4}$ and defining the expansion in powers for the parameter $\beta$ in the form $\beta^{2}=\beta_{0}^{2}+\varepsilon \beta_{1}^{2}$, identifying, the following relation is obtained between the terms of different order for $\beta$ and $\omega$

$$
\frac{\rho_{p} h}{D} \omega_{0}^{2}=\beta_{0}^{4}, \quad \frac{\rho_{p} h}{D} \omega_{0} \omega_{1}=\beta_{0}^{2} \beta_{1}^{2} .
$$

Collecting terms of order $\varepsilon^{0}$ and of order $\varepsilon^{1}$, the next equations are obtained: For the order of $\varepsilon^{0}$,

$$
\begin{aligned}
D \nabla^{4} \tilde{w}_{0}(r, \theta)-\beta_{0}^{4} \tilde{w}_{0}(r, \theta) & =0 \\
\tilde{w}_{0}(a, \theta) & =0 \\
\frac{\partial \tilde{w}_{0}(a, \theta)}{\partial r} & =0 .
\end{aligned}
$$

For the order of $\varepsilon^{1}$,

$$
\begin{gathered}
D \nabla^{4} \tilde{w}_{1}(r, \theta)-\beta_{0}^{4} \tilde{w}_{1}(r, \theta)=2 \beta_{0}^{2} \beta_{1}^{2} \tilde{w}_{0}, \\
\tilde{w}_{1}(a, \theta)+\frac{\partial \tilde{w}_{0}(a, \theta)}{\partial r} f(\theta)=0, \\
\frac{\partial \tilde{w}_{1}(a, \theta)}{\partial r}-\frac{1}{a^{2}} f^{\prime}(\theta) \frac{\partial \tilde{w}_{0}(a, \theta)}{\partial \theta}+f(\theta) \frac{\partial^{2} \tilde{w}_{0}(a, \theta)}{\partial r^{2}}=0 .
\end{gathered}
$$

It is common practice to search for the solution of the zero-order formulation by separation of variables:

$$
\tilde{w}_{0}(r, \theta)=\sum_{0}^{\infty} W_{0 m}^{n}(r)\left\{\begin{array}{l}
\cos m \theta \\
\sin m \theta
\end{array}\right.
$$

where $m$ is the index for the number of nodal diameters, with $m=0$ meaning no nodal diameters, $m=1$ meaning one nodal diameter and so on. 
The zero-order solution is (see Leissa [1969])

$$
W_{0 m}^{n}(r)=A_{0 m}^{n} J_{m}\left(\beta_{0 m}^{n} r\right)+B_{0 m}^{n} I_{m}\left(\beta_{0 m}^{n} r\right) .
$$

Applying the first boundary condition yields

$$
W_{0 m}^{n}(r)=A_{0 m}^{n}\left[J_{m}\left(\beta_{0 m}^{n} r\right)-\lambda_{0 m}^{n} I_{m}\left(\beta_{0 m}^{n} r\right)\right] .
$$

With

$$
\lambda_{0 m}^{n}=\frac{J_{m}\left(\beta_{0 m}^{n} a\right)}{I_{m}\left(\beta_{0 m}^{n} a\right)} .
$$

The parameter $\beta_{0 m}^{n}$, where $\beta_{0 m}^{n}{ }^{4}=\omega_{0 m}^{n}{ }^{2} \frac{\rho_{p} h}{D}$, are the roots of the equation obtained from the second boundary condition

$$
J_{m-1}\left(\beta_{0 m}^{n} a\right)-J_{m+1}\left(\beta_{0 m}^{n} a\right)-\frac{J_{m}\left(\beta_{0 m}^{n} a\right)}{I_{m}\left(\beta_{0 m}^{n} a\right)}\left[I_{m-1}\left(\beta_{0 m}^{n} a\right)+I_{m+1}\left(\beta_{0 m}^{n} a\right)\right]=0,
$$

where $J_{m}$ and $I_{m}$ are the Bessel and the modified Bessel function of the $m$ th order.

For the order $\varepsilon$, the function $\tilde{w}_{1}(r, \theta)$ can also be expressed as

$$
\tilde{w}_{1}(r, \theta)=\sum_{m=0}^{\infty} W_{1 m}^{n}(r)\left\{\begin{array}{l}
\cos m \theta \\
\sin m \theta
\end{array}\right.
$$

The solution to the order $\varepsilon^{1}$ for $W_{1 m}^{n}$, Eq. (2.12), can be obtained as a solution to the homogeneous equation and a particular solution. Thus,

$$
W_{1 m}^{n}(r)=W_{1 m h}^{n}(r)+W_{1 m p}^{n}(r),
$$

where the homogeneous solution is expressed as

$$
W_{1 m h}^{n}(r)=A_{1 m}^{n} J_{m}\left(\beta_{0 m}^{n} r\right)+B_{1 m}^{n} I_{m}\left(\beta_{0 m}^{n} r\right) .
$$

After substitution in Eq. (2.12), the following differential equations for the particular solution are obtained for the cases $m=0$ and $m=1$.

$$
\begin{aligned}
& \frac{d^{4} W_{10 p}^{n}}{d r^{4}}+\frac{2}{r} \frac{d^{3} W_{10 p}^{n}}{d r^{3}}-\frac{1}{r^{2}} \frac{d^{2} W_{10 p}^{n}}{d r^{2}}+\frac{1}{r^{3}} \frac{d W_{10 p}^{n}}{d r}-\beta_{00}^{n}{ }^{4} W_{10 p}^{n} \\
& \quad=2 \beta_{00}^{n}{ }^{2} \beta_{10}^{n}{ }^{2} W_{00}^{n} \\
& \frac{d^{4} W_{11 p}^{n}}{d r^{4}}+\frac{2}{r} \frac{d^{3} W_{11 p}^{n}}{d r^{3}}-\frac{3}{r^{2}} \frac{d^{2} W_{11 p}^{n}}{d r^{2}}+\frac{3}{r^{3}} \frac{d W_{11 p}^{n}}{d r}-\frac{3}{r^{4}} W_{11 p}^{n}-\beta_{01}^{n}{ }^{4} W_{11 p}^{n} \\
& =2 \beta_{01}^{n}{ }^{2} \beta_{11}^{n}{ }^{2} W_{01}^{n} .
\end{aligned}
$$

If the plate shape is assumed to be almost elliptical, the function $f(\theta)$ can be approximated by $f(\theta) \cong-\frac{a}{4}(1-\cos 2 \theta)$.

The boundary conditions for $W_{10}^{n}$ and $W_{11}^{n}$ are now

$$
\begin{aligned}
W_{10}^{n}(r) & =0 & \text { at } r & =a, \\
\frac{d W_{10}^{n}}{d r} & =\frac{a}{4} \frac{d^{2} W_{00}^{n}}{d r^{2}}-\frac{a}{8} \frac{d^{2} W_{02}^{n}}{d r^{2}} & \text { at } r & =a .
\end{aligned}
$$


And

$$
\begin{aligned}
W_{11}^{n}(r) & =0 & \text { at } r & =a, \\
\frac{d W_{11}^{n}}{d r} & =\frac{a}{8} \frac{d^{2} W_{01}^{n}}{d r^{2}}-\frac{a}{8} \frac{d^{2} W_{03}^{n}}{d r^{2}} & \text { at } r & =a .
\end{aligned}
$$

The particular solution $W_{1 m p}^{n}(r)$ is obtained by expansion of the inhomogeneous term in Eqs. (2.21) and (2.22) as a power series:

$$
\frac{W_{1 m p}^{n}(r)}{A_{0 m}^{n}}=\sum_{j=0}^{N}\left[b_{j 0}+b_{j 1} \cdot \beta_{1 m}^{n}{ }^{2}\right] \cdot r^{j} .
$$

With the coefficients $b_{j 0}$ and $b_{j 1}$ known functions of $\lambda_{0 m}^{n}$ and the powers of $\beta_{0 m}^{n}$

$$
b_{j 0}=b_{j 0}\left[\left(\beta_{0 m}^{n}\right)^{j}\right], \quad b_{j 1}=b_{j 1}\left[\left(\beta_{0 m}^{n}\right)^{j-2}, \lambda_{0 m}^{n}\right] .
$$

So that, the particular solution at $r=a$ can be expressed as a function $\Psi$ of the parameters $\beta_{0 m}^{n} a, \lambda_{0 m}^{n}$ and the unknown parameter $\beta_{1 m}^{n} a$, that is

$$
\frac{W_{1 m p}^{n}(a)}{A_{0 m}^{n}}=\Psi\left(\beta_{0 m}^{n} a, \beta_{1 m}^{n} a, \lambda_{0 m}^{n}\right) .
$$

$N$ is taken large enough to ensure convergence.

From the boundary condition $W_{1 m}^{n}(a)=0$ and equating $A_{1 m}^{n}=A_{0 m}^{n}$ the following expression is obtained:

$$
B_{1 m}^{n}=-\frac{A_{0 m}^{n} J_{m}\left(\beta_{0 m}^{n} a\right)+A_{0 m}^{n} \sum_{j=0}^{N}\left[b_{j 0}+b_{j 1} \cdot \beta_{1 m}^{n}{ }^{2}\right] \cdot a^{j}}{I_{m}\left(\beta_{0 m}^{n} a\right)} .
$$

So the homogeneous solution is expressed in the form

$$
W_{1 m h}^{n}(r)=A_{0 m}^{n}\left[J_{m}\left(\beta_{0 m}^{n} r\right)-\lambda_{1 m}^{n} I_{m}\left(\beta_{0 m}^{n} r\right)\right]
$$

with the parameter

$$
\lambda_{1 m}^{n}=\frac{1}{I_{m}\left(\beta_{0 m}^{n} a\right)}\left[J_{m}\left(\beta_{0 m}^{n} a\right)+\Psi\left(\beta_{0 m}^{n} a, \beta_{1 m}^{n} a, \lambda_{0 m}^{n}\right)\right] .
$$

In this way the parameter $\lambda_{1 m}^{n}$ is a known function $\Lambda$ of the parameters $\beta_{0 m}^{n} a$, $\lambda_{0 m}^{n}$ and the unknown parameter $\beta_{1 m}^{n} a$, that is:

$$
\lambda_{1 m}^{n}=\Lambda\left(\beta_{0 m}^{n} a, \beta_{1 m}^{n} a, \lambda_{0 m}^{n}\right) .
$$

Now, from the other boundary condition, a characteristic equation is obtained to determine the unknown value of $\beta_{1 m}^{n} a$ and with the relation $\frac{\rho_{p} h}{D} \omega_{0 m}^{n} \omega_{1 m}^{n}=$ $\beta_{0 m}^{n}{ }^{2} \beta_{1 m}^{n}{ }^{2}$, Eq. (2.10), the perturbed frequency $\omega_{1 m}^{n}$ can be obtained. In a general form, the values for $\beta_{1 m}^{n} a$ depend on the homogeneous solution to the differential equation, the particular solution and Bessel functions of the parameter $\beta_{0 m}^{n} a$ and can be expressed as a function $\Upsilon$

$$
\beta_{1 m}^{n} a=\Upsilon\left[\beta_{0 m}^{n} a, J_{\alpha}\left(\beta_{0 m}^{n} a\right), I_{\alpha}\left(\beta_{0 m}^{n} a\right)\right]
$$

with $\alpha$ taking values $0,1,2$. 
Table 1. Comparison of the fundamental frequency between the present method and other approximated expressions given in Leissa [1969] as function of the eccentricity of the plate.

\begin{tabular}{lcccccc}
\hline$a / b$ & $\varepsilon$ & $\begin{array}{c}\text { Present } \\
\text { method }\end{array}$ & $\begin{array}{c}\text { Leissa [1969] } \\
\text { Eq. }(3.2)\end{array}$ & $\begin{array}{c}\text { Leissa [1969] } \\
\text { Eq. }\left(3.4^{*}\right)\end{array}$ & $\begin{array}{c}\text { Leissa [1969] } \\
\text { Eq. (3.6) }\end{array}$ & $\begin{array}{c}\text { Leissa [1969] } \\
\text { Eq. (3.8) }\end{array}$ \\
\hline 1.000 & 0.00 & 3.1962 & 3.1961 & 3.1961 & 3.2137 & 3.1979 \\
1.02598 & 0.05 & 3.2359 & 3.2380 & 3.2361 & 3.2560 & 3.2400 \\
1.05409 & 0.10 & 3.2751 & 3.2839 & 3.2760 & 3.3029 & 3.2867 \\
1.08465 & 0.15 & 3.3139 & 3.3337 & 3.3160 & 3.3552 & 3.3387 \\
1.11803 & 0.20 & 3.3522 & 3.3875 & 3.3560 & 3.4139 & 3.3971 \\
\hline
\end{tabular}

For the lowest frequency, the following results have been obtained:

$$
\beta_{00}^{0}=3.1962 \text { and } \beta_{10}^{0}=2.2601 .
$$

In Table 1, the values of the parameter $\lambda$ are defined as

$$
\lambda=\left(\frac{\rho_{p} \omega^{2}}{D}\right)^{\frac{1}{4}} a .
$$

In this table, the lowest frequency is compared with three different approximations reported in Leissa [1969], namely, expressions (3.2), (3.6) and (3.8). Expression $\left(3.4^{*}\right)$ is like (3.2) but with only linear terms in $\varepsilon$. It should be noted that (3.2), (3.6) and (3.8) retain terms up to order $\varepsilon^{2}$.

It can be seen that, for the lowest frequency, the comparison between the present method and $\left(3.4^{*}\right)$ is very good.

Next, the natural frequencies for the plate in vacuum are compared with the methods presented in Kaplunov et al. [2005].

For the values of $\frac{a}{b}=1.1, \varepsilon=0.181818$ and $\eta=\frac{h / 2}{a}=0.05$, the frequency parameter defined in Kaplunov et al. [2005] as $\Omega=\frac{\omega a}{\sqrt{\frac{E}{2(1+\nu) \rho_{P}}}}$ is compared for different nodal diameters " $m$ " and nodal circles " $n$ " in Table 2.

It can be observed that the results are quite similar, except for the case of $m=1$ and $n=1$, however, for this case the comparison of the present method with the circular plate, $\varepsilon=0$, seems to be in accordance in contrast with the results obtained by Kaplunov et al. [2005].

In Table 3 , the parameters $\beta_{\varepsilon m}^{n}$ with no nodal diameter $m=0$ and one nodal diameter $m=1$ are presented.

Table 2. Comparison of the natural frequencies of an elliptic plate with $a / b=1.1$.

\begin{tabular}{cccccc}
\hline$m$ & $n$ & $\begin{array}{c}\text { Rayleight-Ritz } \\
\text { Kaplunov et al. }[2005]\end{array}$ & $\begin{array}{c}\text { Present } \\
\text { method }\end{array}$ & $\begin{array}{c}\text { 3D finite element } \\
\text { Kaplunov et al. [2005] }\end{array}$ & $\begin{array}{c}\text { Circular } \\
\text { plate } \varepsilon=0\end{array}$ \\
\hline 0 & 0 & 0.5519 & 0.5437 & 0.538 & 0.4983 \\
1 & 0 & 1.093 & 1.0844 & 1.04 & 1.037 \\
0 & 1 & 2.200 & 2.1165 & 2.01 & 1.94 \\
1 & 1 & 2.707 & 3.096 & 2.43 & 2.967 \\
\hline
\end{tabular}


Table 3. Frequency parameters of orders $\varepsilon^{0}$ and $\varepsilon^{1}$ for the elliptical clamped plate.

\begin{tabular}{ccccc}
\hline$n$ & $\beta_{00}^{n}$ & $\beta_{10}^{n}$ & $\beta_{01}^{n}$ & $\beta_{11}^{n}$ \\
\hline 0 & 3.1962 & 2.2601 & 4.6109 & 2.3054 \\
1 & 6.3064 & 4.4537 & 7.7903 & 3.8997 \\
2 & 9.4305 & 6.6747 & 10.9581 & 5.4779 \\
3 & 12.5771 & 8.8935 & 14.1086 & 7.3482 \\
\hline
\end{tabular}

From these results, the frequencies for different values of eccentricity $\varepsilon$ can be computed.

The advantage of the present method is that now vibration mode is obtained in an explicit form and the method of Amabili and Kwak [1996] can be used to obtain the nondimensional added virtual mass increment NAVMI factors.

\section{NAVMI Factors for Elliptical Plates}

The elliptical plate is assumed to be in contact with an incompressible and inviscid liquid initially at rest. Figure 1 represents an elliptic plate placed into an elliptic hole with an infinite rigid wall and in contract with a liquid.

If the motion of the liquid due to the vibration of the plate is assumed to be irrotational, a velocity potential exists, and the differential equation that describes the fluid motion is the Laplacian equation. If the vibration mode of the plate is expressed as $w(r, \theta, t)=\tilde{w}(r, \theta) \cdot e^{i \omega t}$, the velocity potential can be represented by $\Phi(r, \theta, z, t)=\varphi(r, \theta, z) \cdot i \omega \cdot e^{i \omega t}=\varphi(r, \theta, z) \dot{f}(t)$. The potential $\varphi(r, \theta, z)$ verifies the Laplace equation. The boundary conditions on the velocity potential $\varphi(r, \theta, z)$ are

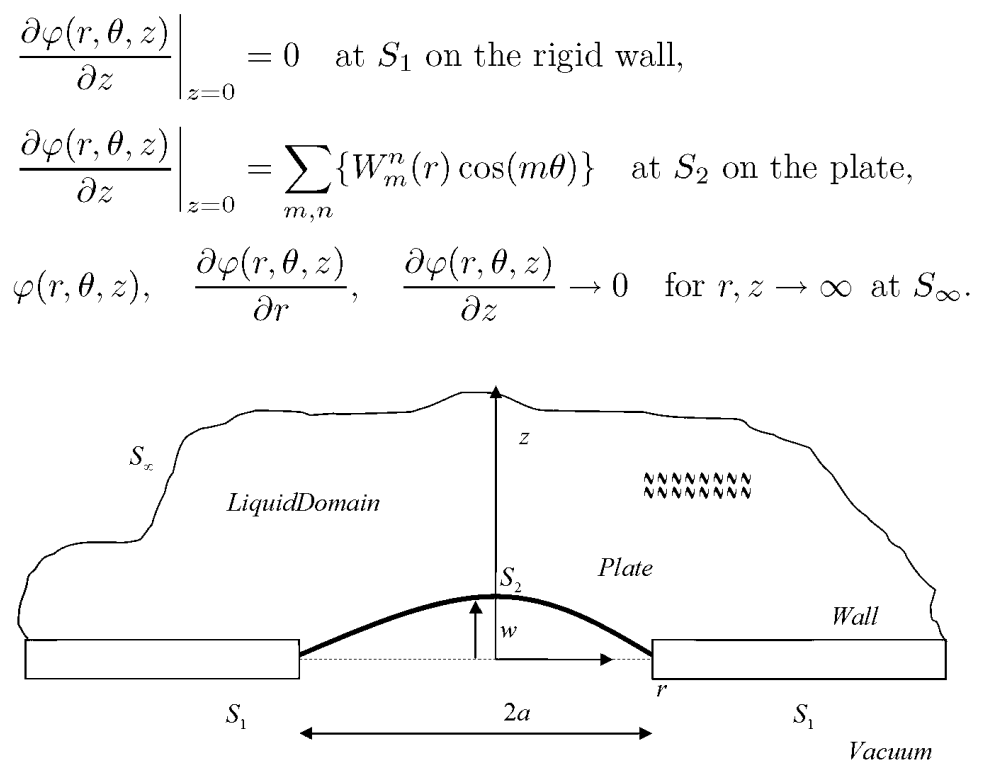

Fig. 1. Elliptic plate placed into an elliptic hole with an infinite rigid wall and in contact with a liquid. 
Following the separation of variables used for the plate motion also the fluid velocity potential can be expressed as

$$
\varphi(r, \theta, z)=\sum_{m, n}\left\{\phi_{m}^{n}(r, z) \cdot \cos (m \theta)\right\}
$$

For each mode of vibration of the plate $W_{m}^{n}(r)$, there will be associated a potential function $\phi_{m}^{n}(r, z)$. The potential $\phi_{m}^{n}$ verifies the following equation in the liquid domain:

$$
\frac{\partial^{2} \phi_{m}^{n}}{\partial r^{2}}+\frac{1}{r} \frac{\partial \phi_{m}^{n}}{\partial r}+\frac{\partial^{2} \phi_{m}^{n}}{\partial z^{2}}-\frac{m^{2}}{r^{2}} \phi_{m}^{n}=0 \quad \text { at } L
$$

Following the procedure used in Amabili and Kwak [1996] for circular plates, the Hankel transformation defined as

$$
\phi_{H m}^{n}(\xi, z)=\int_{0}^{\infty} r \phi_{m}^{n}(r, z) J_{m}(\xi r) d r
$$

is applied to Eq. (3.3). The resulting differential equation for $\phi_{H m}^{n}$ is:

$$
\frac{d^{2} \phi_{H m}^{n}}{d z^{2}}-\xi^{2} \phi_{H m}^{n}=0 .
$$

To satisfy boundary condition equation (3.1c), the potential must decrease with $z$, so the solution to Eq. (3.5) has to be of the form:

$$
\phi_{H m}^{n}(\xi, z)=\Psi_{m}^{n}(\xi) \cdot e^{-\xi z}, \quad \text { for } z \geq 0,
$$

where $\Psi_{m}^{n}$ is a function to be determined from the other boundary conditions. By the inverse Hankel transformation the potential $\phi_{m}^{n}(r, z)$ can be expressed as:

$$
\phi_{m}^{n}(r, z)=\int_{0}^{\infty} \xi \phi_{H m}^{n}(\xi, z) J_{m}(\xi r) d \xi
$$

Now, applying the separation of variables, Eq. (3.2), to the boundary conditions equations (3.1a) and (3.1b) and using Eq. (3.7) yields:

$$
\begin{aligned}
& \sum_{m, n}\left\{\int_{0}^{\infty} \xi^{2} \Psi_{m}^{n}(a \xi) J_{m}(\xi r) \cos (m \theta) d \xi\right\}=\sum_{m, n}\left\{-W_{m}^{n}(r) \cos (m \theta)\right\} \quad \text { at } S_{2}, \\
& \sum_{m, n}\left\{\int_{0}^{\infty} \xi^{2} \Psi_{m}^{n}(a \xi) J_{m}(\xi r) \cos (m \theta) d \xi\right\}=0 \quad \text { at } S_{1}
\end{aligned}
$$

Based on the properties of the Hankel transformation see Sneddon [1966] the solution to Eqs. (3.8a) and (3.8b) is

$$
\sum_{m, n}\left\{\xi \Psi_{m}^{n}(\xi) \cos (m \theta)\right\}=\sum_{m, n}\left\{-\int_{0}^{r_{e}} r W_{m}^{n}(r) \cos (m \theta) J_{m}(r \xi) d r\right\}
$$

where

$$
r_{e}=a\left(1-\frac{\varepsilon}{4}(1-\cos 2 \theta)\right)
$$


After expansion of Eq. (3.9) in powers of $\varepsilon$ and taking into account that $W_{m}^{n}(a)=$ 0 , the expression for $\xi \Psi_{m}^{n}(\xi)$ is

$$
\xi \Psi_{m}^{n}(\xi)=-\int_{0}^{a} r W_{m}^{n}(r) J_{m}(r \xi) d r
$$

From Eq. (3.7) the value of $\phi_{m}^{n}(r, 0)$ is obtained as:

$$
\phi_{m}^{n}(r, 0)=-\int_{0}^{\infty}\left[\int_{0}^{a} \sigma W_{m}^{n}(\sigma) J_{m}(\sigma \xi) d \sigma\right] J_{m}(\xi r) d \xi
$$

If the function $H_{m}^{n}(\xi)$ is defined as

$$
H_{m}^{n}(\xi)=\int_{0}^{a} \sigma W_{m}^{n}(\sigma) J_{m}(\sigma \xi) d \sigma
$$

The final expression for the $m n$ component of the velocity potential at $z=0$ is

$$
\phi_{m}^{n}(r, 0)=-\int_{0}^{\infty} H_{m}^{n}(\xi) J_{m}(\xi r) d \xi .
$$

If it is assumed that the modes for the plate in the liquid are the same as the normal modes in vacuum (there is no coupling see Amabili and Kwak [1996], then each frequency of the plate in the presence of the liquid can be expressed as the ratio to the frequency in vacuum:

$$
\frac{\left(f_{L}\right)_{m}^{n}}{\left(f_{v}\right)_{m}^{n}}=\frac{1}{\sqrt{1+\frac{\left(T_{L}\right)_{m}^{n}}{\left(T_{P}\right)_{m}^{n}}}}=\frac{1}{\sqrt{1+\gamma_{m}^{n}}}
$$

where $\left(T_{L}\right)_{m}^{n}$ and $\left(T_{p}\right)_{m}^{n}$ are the kinetic energies of the liquid and the plate associated to the deformation mode $W_{m}^{n}(r)$.

The ratio of the kinetic energies $\gamma_{m}^{n}$ can be expressed as

$$
\gamma_{m}^{n}=\frac{\rho_{\infty} a}{\rho_{P} h} \Gamma_{m}^{n}
$$

where $\Gamma_{m}^{n}$ is the NAVMI factor and can be expressed as a linear function of $\varepsilon$.

\subsection{Kinetic energy of the liquid associated to the mode $m n\left(T_{L}\right)_{m}^{n}$}

After application of the Gauss theorem, the kinetic energy for the liquid can be expressed as

$$
T_{L}=\frac{1}{2} \rho_{\infty} \iint_{\Sigma} \Phi \frac{\partial \Phi}{\partial n} d \sigma
$$

where $\Sigma=S_{\infty}+S_{1}+S_{2}$. Because $S_{1}$ is a rigid wall, $\frac{\partial \Phi}{\partial n}=-\frac{\partial \Phi}{\partial z}=0$; therefore this surface does not contribute to the kinetic energy. In addition, at $S_{\infty}, \Phi$ and $\frac{\partial \Phi}{\partial n} \rightarrow 0$, so $S_{\infty}$ does not contribute either. Thus, the kinetic energy of the fluid is expressed as

$$
T_{L}=-\left.\frac{1}{2} \rho_{\infty} \int_{0}^{2 \pi} \int_{0}^{r_{e}} \Phi(r, 0, \theta, t) \frac{\partial \Phi}{\partial z}\right|_{z=0} r d r d \theta
$$


The component $m n$ for the kinetic energy can be expressed, after substitution of the velocity potential, as

$$
\left(T_{L}\right)_{m}^{n}=-\left.\frac{1}{2} \rho_{\infty} \dot{f}^{2}(t) \int_{0}^{2 \pi} \int_{0}^{r_{e}} \phi_{m}^{n}(r, 0) \frac{\partial \phi_{m}^{n}}{\partial z}\right|_{z=0} \cos ^{2}(m \theta) r d r d \theta .
$$

However, from the boundary condition, Eq. (3.1b), for $0 \leq r \leq r_{e},\left.\frac{\partial \phi_{m}^{n}}{\partial z}\right|_{z=0}=$ $W_{m}^{n}(r)$; therefore, $\mathrm{Eq} .(3.18)$ is

$$
\left(T_{L}\right)_{m}^{n}=-\frac{1}{2} \rho_{\infty} \dot{f}^{2}(t) \int_{0}^{2 \pi} \int_{0}^{r_{e}} \phi_{m}^{n}(r, 0) W_{m}^{n}(r) \cos ^{2}(m \theta) r d r d \theta .
$$

After expansion in powers of $\varepsilon$, taking into account that $W_{m}^{n}(a)=0$ and using Eq. (3.13) the component $m n$ for the kinetic energy of the fluid can be expressed as

$$
\left(T_{L}\right)_{m}^{n}=\frac{1}{2} \rho_{\infty} \dot{f}^{2}(t) \psi_{m} \int_{0}^{a}\left\{\int_{0}^{\infty} H_{m}^{n}(\xi) J_{m}(\xi r) d \xi\right\} W_{m}^{n}(r) r d r
$$

where

$$
\psi_{m}=\int_{0}^{2 \pi} \cos ^{2} m \theta d \theta= \begin{cases}2 \pi & m=0 \\ \pi & m \neq 0\end{cases}
$$

Interchanging the order of integration, this kinetic energy can also be expressed as

$$
\left(T_{L}\right)_{m}^{n}=\frac{1}{2} \rho_{\infty} \dot{f}^{2}(t) \psi_{m} \int_{0}^{\infty} H_{m}^{n 2}(\xi) d \xi
$$

The function $H_{m}^{n}(\xi)$ depends on the deformation mode $W_{m}^{n}(\xi)$ as

$$
H_{m}^{n}(\xi)=H_{0 m}^{n}(\xi)+\varepsilon H_{1 m}^{n}(\xi)=\int_{0}^{a}\left(W_{0 m}^{n}(\sigma)+\varepsilon W_{1 m}^{n}(\sigma)\right) J_{m}(\sigma \xi) \sigma d \sigma
$$

Taking dimensionless variables defined as $\rho=\frac{r}{a}, \eta=a \xi$ and naming $\bar{H}_{m}^{n}=\frac{H_{m}^{n}}{a^{2}}$ the expression for $\bar{H}_{0 m}^{n}(\eta)$ considering the deformation mode $W_{0 m}^{n}(\rho)$ [Eq. (2.14)] is:

$$
\bar{H}_{0 m}^{n}(\eta)=\int_{0}^{1} A_{o m}^{n}\left[J_{m}\left(\beta_{0 m}^{n} a \rho\right)-\lambda_{0 m}^{n} I_{m}\left(\beta_{0 m}^{n} a \rho\right)\right] J_{m}(\eta \rho) \rho d \rho .
$$

This integral has an analytic expression, see Bowman [1958]:

$$
\bar{H}_{0 m}^{n}(\eta)=A_{0 m}^{n}\left[\begin{array}{l}
\frac{\eta J_{m-1}(\eta) J_{m}\left(\beta_{0 m}^{n} a\right)-\beta_{0 m}^{n} a J_{m-1}\left(\beta_{0 m}^{n} a\right) J_{m}(\eta)}{\beta_{0 m}^{n}{ }^{2} a^{2}-\eta^{2}} \\
-\lambda_{0 m}^{n} \frac{\eta J_{m+1}(\eta) I_{m}\left(\beta_{0 m}^{n} a\right)+\beta_{0 m}^{n} a I_{m+1}\left(\beta_{0 m}^{n} a\right) J_{m}(\eta)}{\beta_{0 m}^{n}{ }^{2} a^{2}+\eta^{2}}
\end{array}\right] .
$$

The expression for $\bar{H}_{1 m}^{n}(\eta)$ has also two parts one due to the homogeneous solution $W_{1 m h}^{n}(\rho)$ and the other due to the particular solution $W_{1 m p}^{n}(\rho)$, Eq. (2.19), therefore:

$$
\bar{H}_{1 m}^{n}(\eta)=\bar{H}_{1 m h}^{n}(\eta)+\bar{H}_{1 m p}^{n}(\eta) .
$$

The function $\bar{H}_{1 m h}^{n}(\eta)$ has the same expression as Eq. (3.24) replacing $\lambda_{0 m}^{n}$ by $\lambda_{1 m}^{n}$. 
The function $\bar{H}_{1 m p}^{n}(\eta)$ associated to the particular solution, is:

$$
\bar{H}_{1 m p}^{n}(\eta)=\int_{0}^{1} W_{1 m p}^{n}(\rho) J_{m}(\eta \rho) \rho d \rho .
$$

If $W_{1 m p}^{n}(\rho)$ of Eq. (2.25), is expressed as a Dini's expansion [Bowman, 1958]

$$
W_{1 m p}^{n}(\rho)=\sum_{j=0}^{N} A_{j} J_{m}\left(\alpha_{j} \rho\right)
$$

where $N$ is large enough to assure convergence and the coefficients $A_{j}$ and $\alpha_{j}$ are defined as:

$$
A_{j}=\frac{2}{J_{m}^{2}\left(\alpha_{j}\right)-J_{m-1}\left(\alpha_{j}\right) J_{m+1}\left(\alpha_{j}\right)} \int_{0}^{1} \rho W_{1 m p}^{n}(\rho) J_{m}\left(\alpha_{j} \rho\right) d r .
$$

And $\alpha_{j}$ are the roots of equation $J_{m}^{\prime}(\rho)=0$.

Then, $\bar{H}_{1 m p}^{n}(\eta)$ is expressed as:

$$
\bar{H}_{1 m p}^{n}(\eta)=\sum_{j=0}^{N} A_{j} \frac{\eta J_{m-1}(\eta) J_{m}\left(\alpha_{j}\right)-\alpha_{j} J_{m-1}\left(\alpha_{j}\right) J_{m}(\eta)}{\alpha_{j}^{2}-\eta^{2}}
$$

So finally the component $m n$ for the kinetic energy of the liquid for the $m n$ mode is evaluated from the expression

$$
\left(T_{L}\right)_{m}^{n}=\frac{1}{2} \rho_{\infty} \dot{f}^{2}(t) \psi_{m} a^{3} \int_{0}^{\infty} \bar{H}_{m}^{n 2}(\eta) d \eta
$$

where $\bar{H}_{m}^{n}(\eta)$ is expressed as function of $\bar{H}_{0 m}^{n}(\eta)$ and of $\bar{H}_{1 m}^{n}(\eta)$ through Eqs. (3.24) and (3.25). The integral is evaluated numerically.

\subsection{Kinetic energy of the plate, $T_{p}$}

The kinetic energy for the vibrating plate is given by

$$
T_{p}=\frac{1}{2} \rho_{p} h \int_{0}^{r_{e}} \int_{0}^{2 \pi} \dot{w}^{2}(r, \theta, t) r d r d \theta .
$$

The deformation velocity, assuming harmonic motion, is expressed as

$$
\dot{w}(r, \theta, t)=\sum_{m, n}\left\{W_{m}^{n}(r) \cos (m \theta) i \omega e^{i \omega t}\right\}=\sum_{m, n}\left\{W_{m}^{n}(r) \cos (m \theta) \dot{f}(t)\right\} .
$$

By substitution of Eq. (3.32) in Eq. (3.31), the kinetic energy of the plate for the $m n$ mode is

$$
\left(T_{p}\right)_{m}^{n}=\frac{1}{2} \rho_{p} h \dot{f}(t)^{2} \int_{0}^{2 \pi} \int_{0}^{r_{e}} W_{m}^{n 2}(r) \cos ^{2}(m \theta) r d r d \theta .
$$




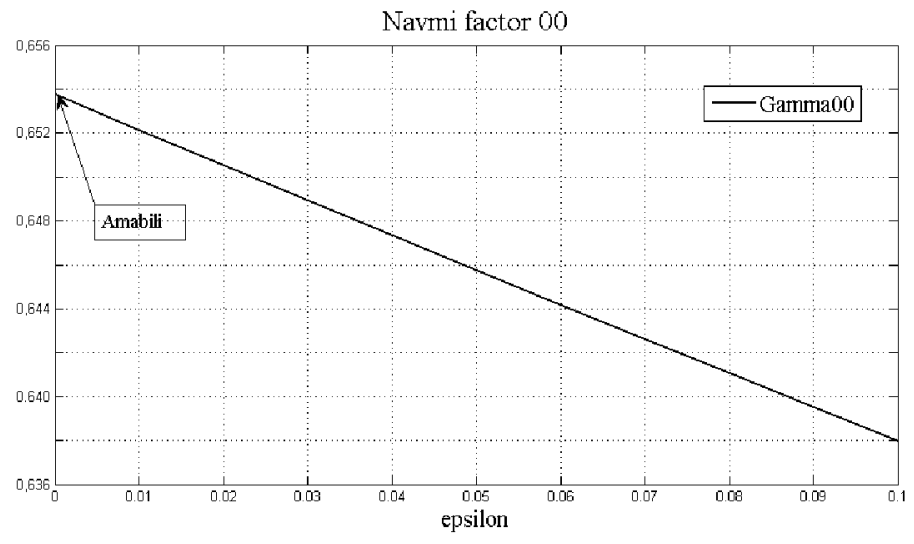

(a)

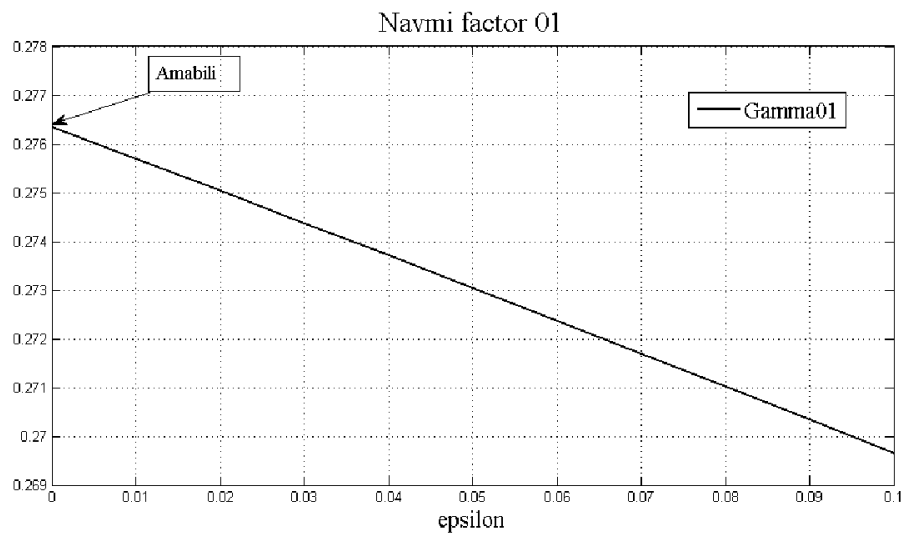

(b)

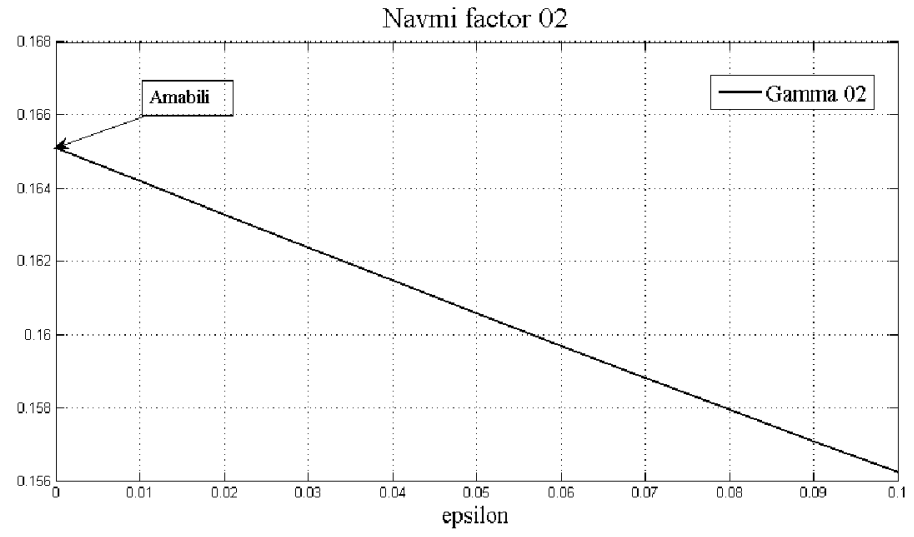

(c)

Fig. 2. (a), (b) and (c): NAVMI factors as a function of eccentricity for the modes without nodal diameters. 


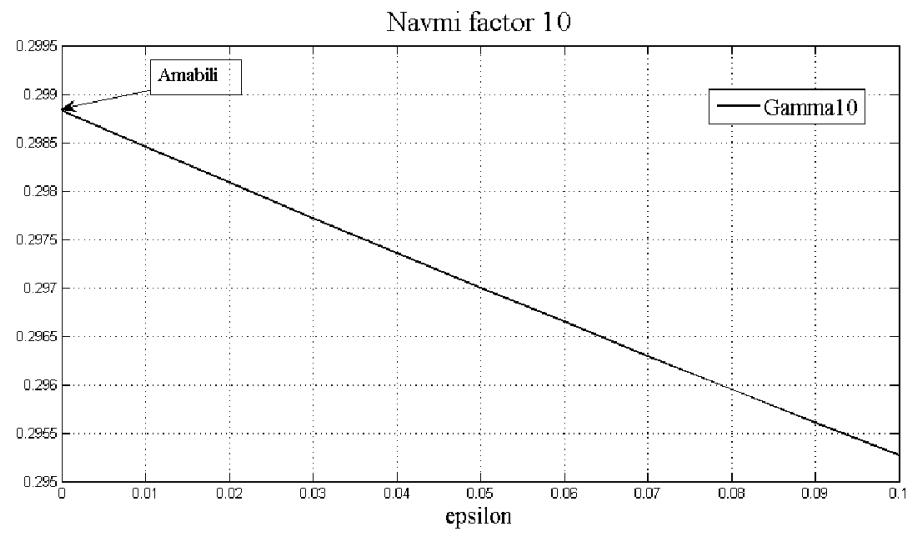

(a)

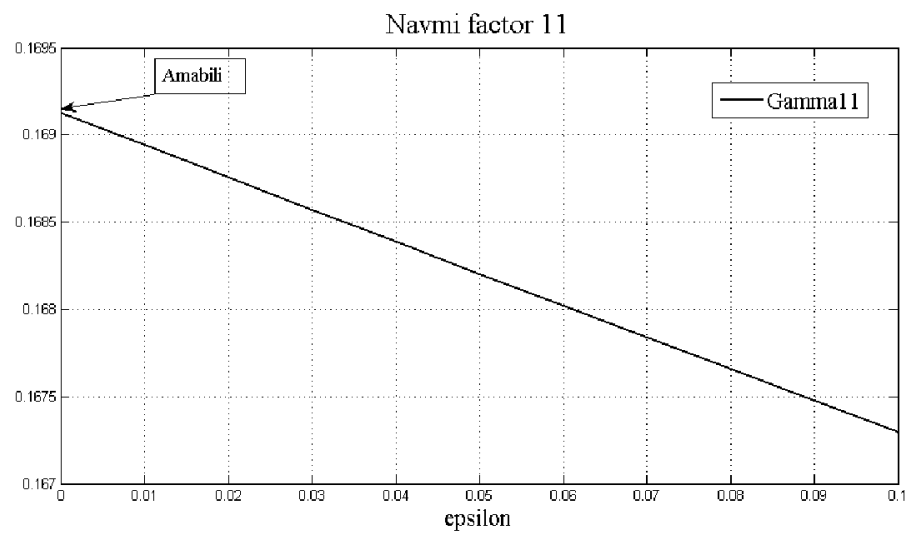

(b)

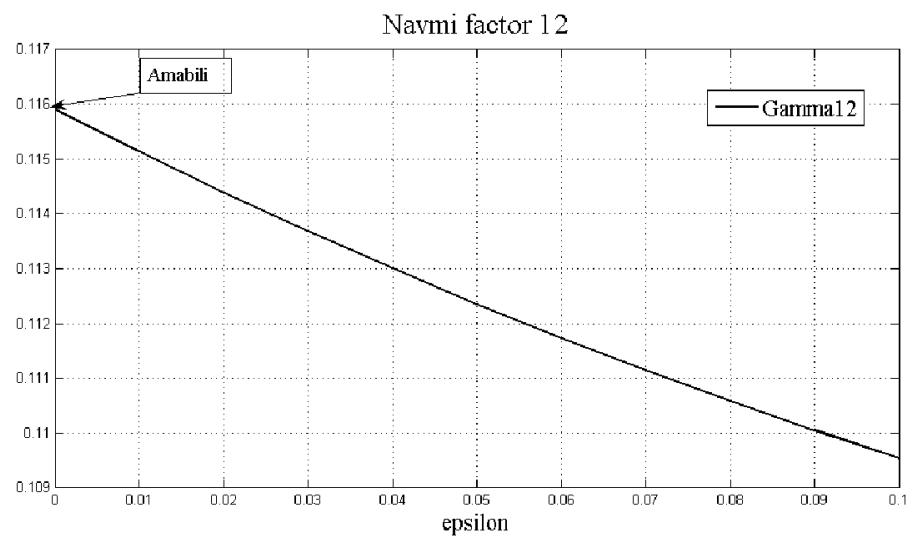

(c)

Fig. 3. (a), (b) and (c): NAVMI factors as a function of eccentricity for the modes with one nodal diameter. 


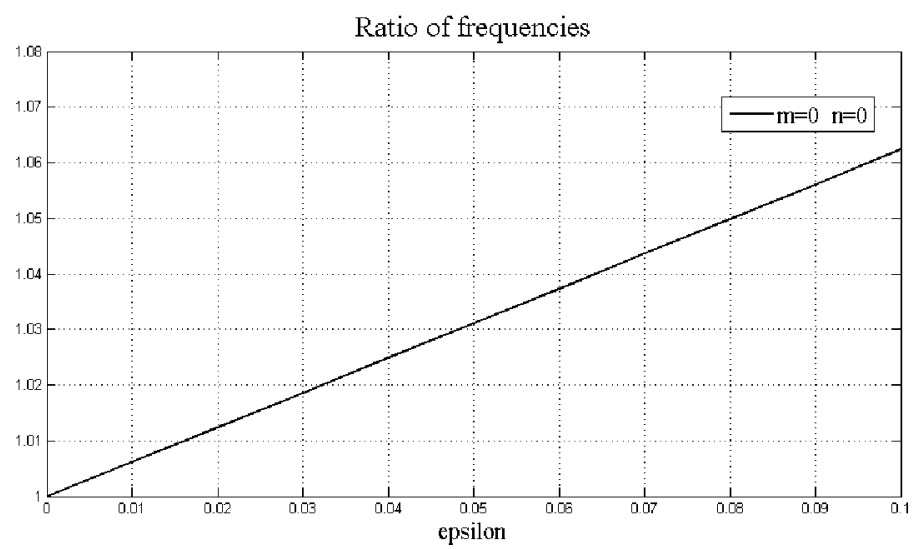

(a)

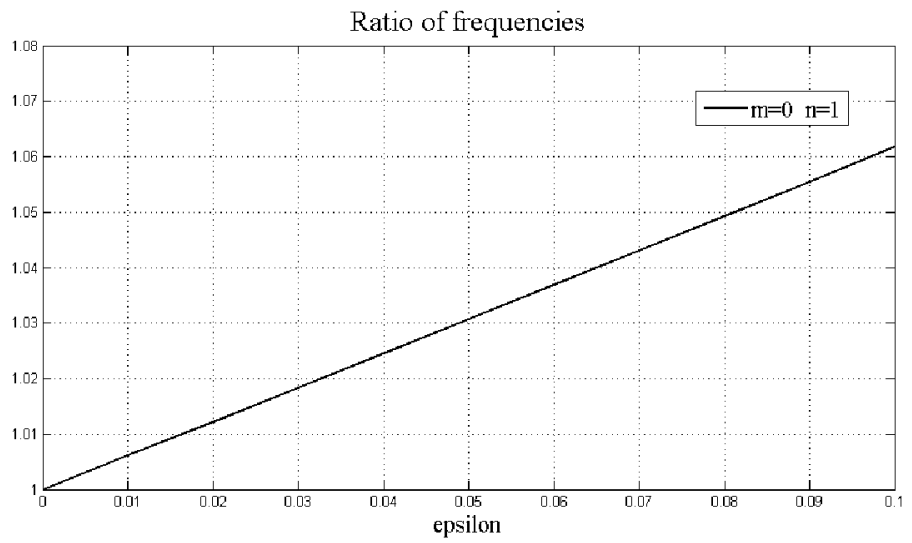

(b)

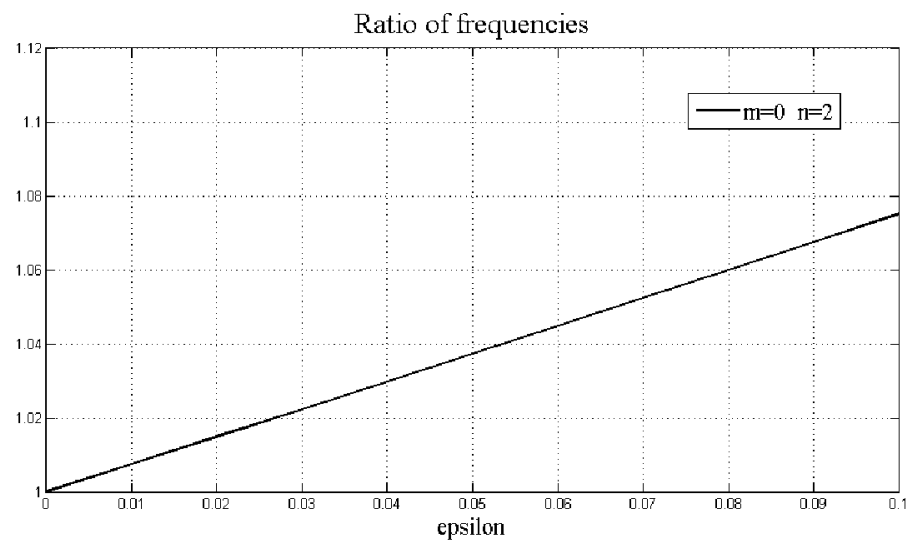

(c)

Fig. 4. (a), (b) and (c): Influence of ellipticity on the natural frequencies of the plate immersed in the liquid for the case of no nodal diameter. 


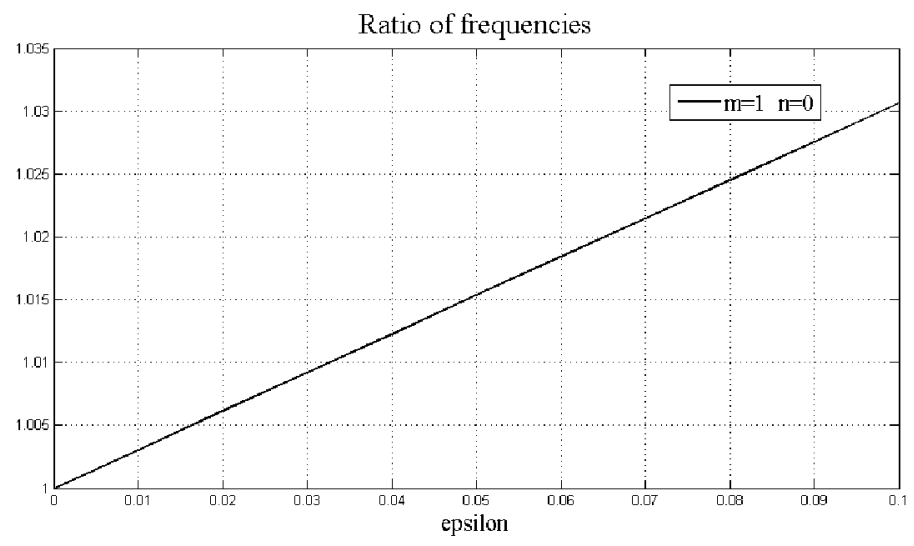

(a)

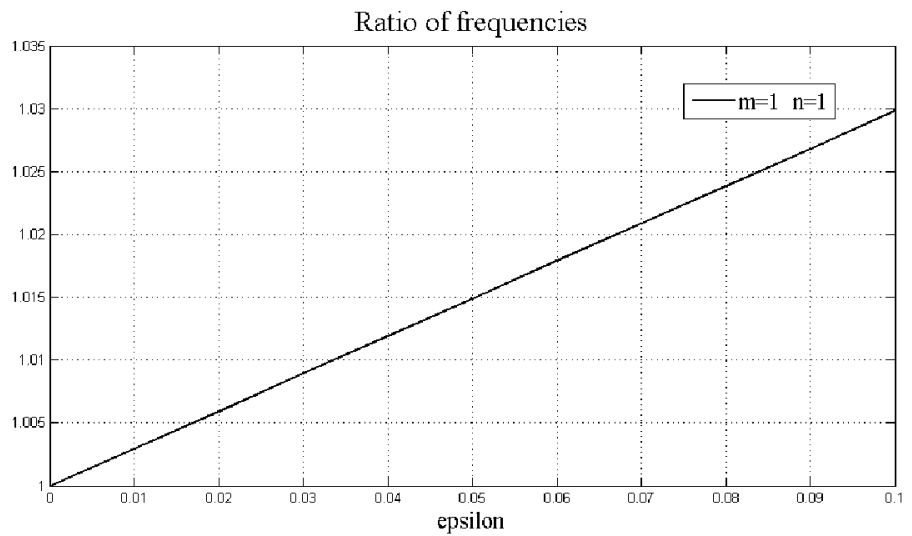

(b)

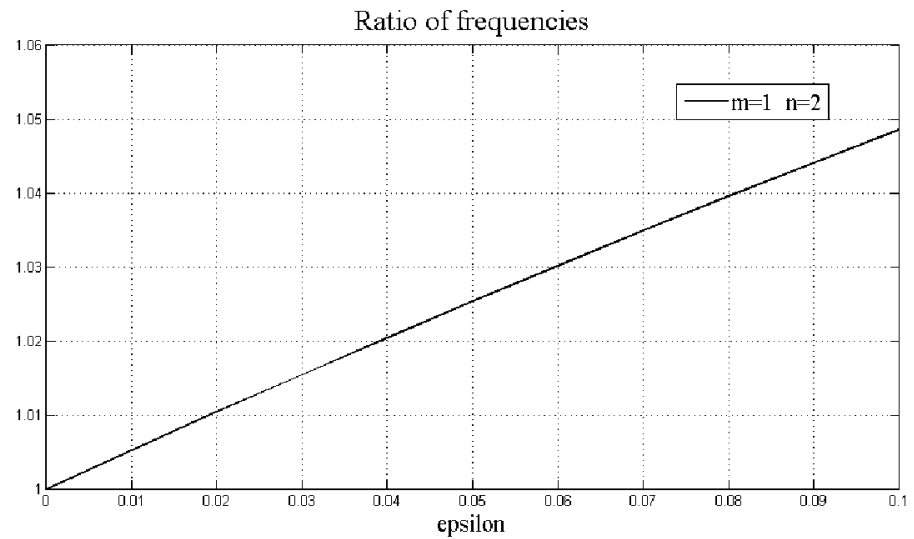

(c)

Fig. 5. (a), (b) and (c): Influence of ellipticity on the natural frequencies of the plate immersed in the liquid for the case of one nodal diameter. 
Expanding in power series of $\varepsilon$ and taking into account that $W_{m}^{n}(a)=0$, the kinetic energy of the plate for the $m n$ mode is

$$
\left(T_{p}\right)_{m}^{n}=\frac{1}{2} \rho_{p} h \dot{f}(t)^{2} \psi_{m} \int_{0}^{a}\left[W_{0 m}^{n}(r)+\varepsilon \cdot W_{1 m}^{n}(r)\right]^{2} r d r .
$$

Taking dimensionless variables $\rho=\frac{r}{a}$ :

$$
\left(T_{p}\right)_{m}^{n}=\frac{1}{2} \rho_{p} h a^{2} \dot{f}(t)^{2} \psi_{m} \int_{0}^{1}\left[W_{0 m}^{n}(\rho)+\varepsilon \cdot W_{1 m}^{n}(\rho)\right]^{2} \rho d \rho .
$$

Next, the results for the cases of $m=0$ and $m=1$ and $n=0,1$ and 2 are presented in Figs. 2 and 3. Also represented is the result for the circular plate obtained by Amabili and Kwak [1996].

From these results the following conclusion can be obtained:

(1) The value of the NAVMI factor always decreases with the eccentricity.

(2) The variation is greater in the modes without a nodal diameter (of the order of $2.5 \%$ ) than for the modes with one nodal diameter (less than $1 \%$ ).

(3) For the same number of nodal diameters, the reduction increases with the order of the mode, that is, with $n$.

Next, the ratio between the frequencies of the elliptic plate and the circular plate in liquid will be computed. This ratio can be expressed as

$$
\frac{\left[\left(f_{L}\right)_{m}^{n}\right]_{\varepsilon}}{\left[\left(f_{L}\right)_{m}^{n}\right]_{0}}=\frac{\left[\left(f_{v}\right)_{m}^{n}\right]_{\varepsilon}}{\left[\left(f_{v}\right)_{m}^{n}\right]_{0}} \sqrt{\frac{1+\left(\gamma_{m}^{n}\right)_{0}}{1+\left(\gamma_{m}^{n}\right)_{\varepsilon}}}
$$

where $\left[\left(f_{L}\right)_{m}^{n}\right]_{\varepsilon}$ represents the natural frequency of the elliptic plate in the liquid, $\left[\left(f_{L}\right)_{m}^{n}\right]_{0}$ the natural frequency of the circular plate in liquid, $\left[\left(f_{v}\right)_{m}^{n}\right]_{\varepsilon}$ is the natural frequency of the elliptic plate in vacuum and $\left[\left(f_{v}\right)_{m}^{n}\right]_{0}$ is the natural frequency of the circular plate in vacuum.

In Figs. 4 and 5, these ratios of frequencies are presented for the cases of $m=0$ and 1 and for $n=0,1$ and 2 .

Again, in the figures, it can be observed that the frequencies that depend linearly on $\varepsilon$ now increase with respect to the case of the circular plate. The increase is faster for the modes without nodal diameters than for those with one nodal diameter, as expected.

\section{Conclusions}

The method of the Hankel transformation, which was first used by Magrab [1979] and later by Amabili and Kwak [1996] for circular plates, is now applied to solve the fluid-structure interaction problem for elliptical plates. For the application of the method, an explicit expression for the vibration mode of the plate is needed. Therefore, for plates with a small eccentricity ratio $\varepsilon$, a perturbation technique has been applied that within the first order of $\varepsilon$ makes it possible to obtain the normal 
modes and natural frequencies of the elliptical plate in vacuum. The results obtained for these cases are shown to be in very good agreement when comparing the natural frequencies with other approximate methods for the same order of accuracy.

For the results with the fluid, the NAVMI factors has been calculated for the cases without nodal diameters and with one nodal diameter. The results show that, for all cases, the influence of ellipticity reduces the NAVMI factor, and the reduction is greater for the modes of higher order for the same value of $m$ and more important, as expected, for the case with zero nodal diameters than for the case with one nodal diameter.

The method can be used as a test case to check numerical codes used based on FEM-BEM to determine the influence of the fluid on the dynamic characteristics of elliptical plates. Also the method could be applied to other plates almost circular by changing the function $f(\theta)$.

\section{References}

Amabili, M. and Kwak, M. K. [1996] "Free vibrations of circular plates coupled with liquids: Revising the Lamb problem," Journal of Fluids and Structures 10, 743-761.

Amabili, M., Pasqualini, A. and Dalpiaz, G. [1995] "Natural frequencies and modes of free-edge circular plates vibrating in vacuum or in contact with liquid," Journal of Sound and Vibration 188, 685-689.

Askari, E., Jeong, K.-H. and Amabili, M. [2013] "Hydroelastic vibration of circular plates immersed in a liquid-filled container with free surface," Journal of Sound and Vibration 332, 3064-3085.

Bowman, F. [1958] Introduction to Bessel Functions (Dover Publications Inc, New York, USA).

Filippi, P. J. T., Lagarrigue, O. and Mattei, P. O. [1994] "Perturbation method for sound radiation by a vibrating plate in a light fluid: Comparison with the exact solution," Journal of Sound and Vibration 177, 259-275.

Geng, Q. and Li, Y. [2012] "Analysis of dynamic and acoustic radiation characters for a flat plate under thermal environments," International Journal of Applied Mechanics $04,16 \mathrm{pp}$.

Kaplunov, J. D., Nolde, E. V. and Shorr, B. F. [2005] "A perturbation approach for evaluating natural frequencies of moderately thick elliptic plates," Journal of Sound and Vibration 281, 905-919.

Kwak, M. K. [1991] "Vibration of circular plates in contact with water," Journal of Applied Mechanics 58, 480-483.

Kwak, M. K. and Kim, K. C. [1991] "Axisymmetric vibration of circular plates in contact with fluid," Journal of Sound and Vibration 146, 381-389.

Lamb, H. [1920] "On the vibrations of an elastic plate in contact with water," Proceedings of the Royal Society of London XCVIII, 205-216.

Leissa, A. W. [1969] Vibration of Plates, NASA SP, Vol. 160 (Scientific and Technical Information Division, NASA).

Magrab, E. B. [1979] Vibration of Elastic Structural Members (Sijthoff and Noordhoff, Germantown, MD).

McLachlan, N. W. [1932] "The accession to inertia of flexible discs vibrating in a fluid," Proceedings of the Royal Society of London 44, 546-555. 
Nayfeh, A. H. [1981] Introduction to Perturbation Techniques (John Wiley and Sons, New York, USA).

Peake, W. H. and Thurston, E. G. [1954] "The lowest resonant frequency of a water-loaded circular plate," Journal of the Acoustical Society of America 26, 166-168.

Rayleigh, J. W. S. [1945] Theory of Sound, 2nd edn. (Dover, New York, USA).

Sneddon, I. N. [1966] Mixed Boundary Value Problems in Potential Theory (North-Holland, Amsterdam, Netherlands).

Tariverdilo, S., Shahmardani, M., Mirzapour, J. and Shabani, R. [2013] "Asymmetric free vibration of circular plate in contact with incompressible fluid," Applied Mathematical Modelling 37, 228-239.

Zheng, H. and Wei, Z. [2013] "Vibroacoustic analysis of stiffened plates with nonuniform boundary conditions," International Journals of Applied Mechanics 5, 19 pp. 